\title{
EL MODELO B-LEARNING IMPLEMENTADO EN LA ASIGNATURA SIMULACIÓN
}

\author{
(B-LEARNING MODEL IMPLEMENTED VIA SIMULATION)
}

\author{
Elena Durán, \\ Rosanna Costaguta \\ Mariela Gola \\ Universidad Católica de Santiago del Estero (Argentina)
}

\section{RESUMEN}

En este artículo se presentan los principales resultados del proyecto de investigación "Una Propuesta de b-learning para la enseñanza de la Simulación”, desarrollado durante los años 2008 y 2009 en la Universidad Católica de Santiago del Estero (UCSE). El objetivo de este proyecto fue elaborar un diseño curricular basado en el modelo B-learning para la asignatura Simulación de la carrera Ingeniería en Computación, y aplicarlo sobre la cohorte 2008 con el fin de evaluar su incidencia en los procesos de enseñanza y de aprendizaje. Dados los resultados positivos obtenidos con esa cohorte, se decidió mejorar la propuesta combinando el modelo b-learning con el aprendizaje colaborativo soportado por computadora, y aplicar esta nueva propuesta a la cohorte 2009.

Palabras clave: blended learning, aprendizaje colaborativo, simulación, educación superior.

\begin{abstract}
This article presents the results of the research project "A b-learning proposal for the teaching of simulation", developed during the years 2008 and 2009 at the Catholic University of Santiago del Estero (UCSE). The aim of the project was to develop a curriculum based on the B-learning model for simulation in Computer Engineering, and to apply it to the 2008 cohort to assess its impact on the teaching-learning process. Due to the positive results obtained with this cohort, the authors improved the proposal combining the b-learning model with computer-supported collaborative learning, and applied it to the 2009 cohort.
\end{abstract}

Keywords: blended learning, collaborative learning, simulation, higher education. 
Sin lugar a dudas muchas son las ventajas que ofrecen las Nuevas Tecnologías de la Información y Comunicación (NTICs) como medios para la educación a distancia. Sin embargo, las NTICs si se usan correctamente pueden generar también grandes beneficios a la educación presencial (Gonzalez, 2006). Estas experiencias de formación combinada son las que reciben el nombre de b-learning (Blended Learning). Se trata de un modelo de enseñanza aprendizaje que incluye tanto formación presencial como e-learning (aprendizaje electrónico), beneficiándose de las ventajas de ambos (López, 2006). Sin embargo, no se trata solo de agregar tecnología a las clases, sino de reemplazar algunas actividades de aprendizaje con otras apoyadas con tecnología (Rosas, 2005). Los sistemas basados en b-learning se caracterizan por la flexibilidad e interactividad que facilitan los recursos de información y las herramientas de comunicación (Chat, foro, correo electrónico, etc.), lo cual permite superar, por ejemplo, los obstáculos que aparecen en el desarrollo de trabajos grupales presenciales (Sánchez, 2005). Obstáculos tales como las limitaciones de tiempo y espacio para las reuniones, el no poder compartir la información simultáneamente, entre otros.

Tal como sugiere Rosas (2005), la idea clave es la selección de los medios adecuados para cada necesidad educativa. Por lo tanto, el b-learning no es simplemente la inclusión de elementos tecnológicos sino la forma en que estos elementos son combinados. Esto significa que para alcanzar los objetivos de aprendizaje esperados la secuencia de elementos combinados es tan importante como los mismos elementos. Es decir, se trata de un modelo ecléctico compuesto por instrucción presencial y funcionalidades del e-learning, con la finalidad de potenciar las fortalezas y disminuir las limitaciones de ambas modalidades (Peña, 2005). Este modelo permite permanecer menos tiempo en el aula, propicia un potencial ahorro de espacio físico, incrementa la participación de los estudiantes como responsables de su propio aprendizaje entre otros beneficios.

En b-learning el formador asume de nuevo su rol tradicional, pero usa en beneficio propio el material didáctico que la Informática e Internet le proporcionan, para ejercer su labor en dos frentes: como tutor on-line (tutorías a distancia) y como educador tradicional (cursos presenciales). La forma en que combine ambas estrategias depende de las necesidades específicas de cada curso. Así, no existe una fórmula única para lograr una solución.

Si bien existen algunas experiencias aisladas de aplicación del modelo b-learning en universidades argentinas (Aballay, 2006; Feierherd, 2005; Kowalski, 2008; Sanz, 2008), son prácticamente nulas en las universidades de Santiago del Estero. 
Por otra parte, en los últimos años el ritmo acelerado y cambiante impone nuevas dinámicas alternativas para acceder y transmitir el conocimiento. El mayor énfasis en la información y la tecnología del mundo laboral actual ha creado la necesidad de formar perfiles profesionales competentes en el manejo de la información y en el empleo de destrezas tecnológicas. En pocos años la mayor parte de la fuerza laboral estará empleada en ocupaciones basadas en el uso informativo (recopilación, proceso de datos, recuperación y análisis de información, etc.). Por lo tanto, es fundamental que los estudiantes adquieran no sólo conocimientos sino también habilidades y procedimientos que vayan a emplear en el mundo laboral, y entre ellos se destaca el desarrollo de competencias en el manejo de información (López, 2006).

La mayor parte de las asignaturas de la carrera de Ingeniería en Computación precisan de un conocimiento que haga posible que el estudiante pueda "aprender haciendo”, no sólo desde el análisis y la asimilación conceptual de las herramientas tecnológicas propias de la disciplina, sino también a través del conocimiento y la comprensión lograda mediante la resolución de ejercicios y prácticas soportadas por las NTICs.

Además, en particular para la asignatura Simulación, el perfil de los estudiantes que la cursan se caracteriza por un cierto grado de madurez académica, ya que se encuentran finalizando la carrera, no muy dados a asistir regularmente a clases, asiduos internautas que en su mayoría poseen acceso a Internet desde sus hogares o lugares de trabajo. Todo esto nos llevó, en una primera experiencia, a desarrollar la alternativa de aprendizaje combinado (b-learning), e implementar la misma en la cohorte 2008.

El modelo b-learning se puede potenciar si se lo combina con el enfoque del Aprendizaje Colaborativo Soportado por Computadora (CSCL - Computer Supported Collaborative Learning), surgido en la década del 90 con el advenimiento de Internet. Un sistema CSCL trata de ofrecer un servicio de mediación que soporta la comunicación y el trabajo de los estudiantes en actividades grupales a través de la red, proporcionando asistencia en su coordinación y aplicación del conocimiento en ciertos dominios (Bartolomé, 2004).

Dados los resultados positivos obtenidos con la cohorte 2008, sumado a la flexibilidad que ofrece la asignatura Simulación para incluir actividades que insten al trabajo colaborativo, así como la posibilidad de inculcarles a los estudiantes el hábito de ahondar de manera autónoma en los conocimientos ofrecido, nos motivó a mejorar la propuesta combinando el modelo b-learning con el aprendizaje 
colaborativo soportado por computadora, propuesta que fue implementada en la cohorte 2009.

Este artículo se organiza como sigue. En primer término se describe la primera experiencia realizada, aplicando el modelo b-learning y se muestran los resultados obtenidos en la misma. A continuación se presenta la experiencia realizada en la que se combina el modelo b-learning con CSCL y se muestran los resultados obtenidos. Finalmente se presentan algunas conclusiones a las que fueron posible llegar con ambas experiencias.

\section{DESCRIPCIÓN DE LA EXPERIENCIA CON EL MODELO B-LEARNING}

La experiencia tuvo como objetivo evaluar la incidencia de la aplicación del modelo b-learning en la calidad de los procesos de enseñanza y de aprendizaje en la asignatura Simulación, correspondiente a la carrera de Ingeniería en Computación de la Universidad Católica de Santiago del Estero. Dicha experiencia se llevó a cabo con la cohorte 2008 de la mencionada asignatura, conformada por 17 estudiantes, y se centró en el abordaje de las tres últimas unidades temáticas del programa de estudio de esta asignatura: Simulación con Dinámica de Sistemas (Unidad 5), Realidad Virtual (Unidad 6) y Nuevas tendencias de la Simulación (Unidad 7). Utilizando la plataforma de e-learning de la universidad, "e-educativa", se llevó a cabo una experiencia de aprendizaje combinado (b-learning). Los estudiantes realizaron cuatro actividades utilizando la plataforma de e-learning y dos actividades presenciales.

Para cumplir con los objetivos de la Unidad 5, los estudiantes asistieron a clases presenciales, en las que los docentes realizaron una introducción al tema y explicaron en detalle la Técnica de Dinámica de Sistemas para desarrollar simulaciones continuas. Luego se planteó a los estudiantes una actividad presencial que fue complementada con otra actividad utilizando la plataforma de e-learning. El objetivo de la actividad presencial fue que los estudiantes apliquen la técnica de Dinámica de Sistemas a la resolución de problemas del mundo real. Para ello desarrollaron un taller construyendo un software para simular una problemática previamente seleccionada. En el marco de esa actividad presencial los estudiantes debían realizar parte del análisis del problema (construcción del diagrama causal) utilizando el foro de la plataforma de e-learning.

Para cumplir con los objetivos de la Unidad 6, los estudiantes asistieron a clases teóricas donde los docentes realizaron una introducción al tema y se plantearon dos 
actividades. La primera tuvo como propósito que los estudiantes se inicien en el conocimiento de los Sistemas de Realidad Virtual: qué son, cómo funcionan y cuáles son las tecnologías que se utilizan; y desarrollen una experiencia en entorno virtual. Para ello se les proporcionó un texto (disponible en la plataforma), que los estudiantes debían leer, para luego discutir cuatro cuestiones planteadas por el docente en el foro habilitado a tal efecto, y acordar las respuestas en un documento. En esta actividad la discusión fue tutorizada por un docente. Finalizada la misma se planteó una segunda actividad con características similares a la primera, con el propósito de reforzar los conocimientos de los estudiantes sobre los Sistemas de Realidad Virtual.

Para cumplir con los objetivos de la Unidad 7, se planteó una actividad presencial bajo la modalidad de Seminario cuyo propósito fue que los estudiantes conocieran las nuevas líneas de investigación en Simulación, desarrollen aptitudes para la investigación, y se iniciaran en el estudio, la interpretación y la crítica de trabajos adelantados en el área de la Simulación. Así, los alumnos conformaron grupos de 3 o 4 integrantes y eligieron el tema de investigación entre la temática propuesta en el programa de la asignatura. Producto de la investigación elaboraron una monografía que fue expuesta en clase. Finalizado el seminario se planteó a los estudiantes una actividad para ser realizada utilizando el foro de la plataforma de e-learning. El propósito de la misma fue que los estudiantes reforzaran sus conocimientos sobre las distintas temáticas abordadas en relación a las nuevas líneas de avance de la Simulación. Para ello los docentes plantearon cinco preguntas a ser debatidas. En forma previa a tales discusiones, los estudiantes debieron leer las monografías elaboradas como resultado del Seminario. Finalizado el plazo para la discusión los estudiantes subieron a la plataforma un documento con el desarrollo de cada una de las respuestas para las preguntas planteadas.

\section{RESULTADOS OBTENIDOS EN LA EXPERIENCIA CON B-LEARNING}

Para evaluar la incidencia del modelo b-learning en los procesos de enseñanza y de aprendizaje se definieron cuatro variables: nivel de conocimientos (NCT), nivel de participación (NPP), nivel de comunicación (NCC) y nivel de producción en trabajos colaborativos (NPC). Para dichas variables se estableció una escala cualitativa con los siguientes valores posibles: alto (A), medio (M) y bajo (B). Además, se diseñó una encuesta que permitió recabar opiniones de los estudiantes una vez finalizada la experiencia.

La variable NCT permitió verificar el grado de apropiación de los temas teóricos y prácticos por parte de los estudiantes. Se consideraron como indicadores los 
resultados de las dos actividades de evaluación llevadas a cabo: parcial (PAR) y taller (TALL). La actividad PAR corresponde a una evaluación de carácter teórico-práctico sobre las unidades temáticas incluidas en la experiencia, donde las calificaciones obtenidas se dividieron en tres rangos: 1 a 4 puntos, 5 a 7 puntos y 8 a 10 puntos. La actividad TALL corresponde a la evaluación del taller de programación sobre Dinámica de Sistemas utilizando el software Evolución (Evolución, 2002). En este caso se consideró una escala cualitativa con las siguientes calificaciones posibles: alto (A), medio (M) y bajo (B). A fin de poder asignar valor a la variable NCT se establecieron relaciones entre ella y los indicadores PAR y TALL (Tabla 1).

La variable NPP permitió evaluar la cantidad de participaciones registradas en los foros electrónicos. Se consideró como indicador la cantidad de participaciones (CP) que los estudiantes efectuaron considerando las cuatro actividades de foro realizadas durante la experiencia. El número de participaciones se dividió en tres rangos: o a 60 participaciones, 61 a 80 participaciones y más de 80 participaciones. A fin de poder asignar valor a la variable NPP se establecieron relaciones entre ella y su indicador (Tabla 1).

La variable NCC permitió evaluar la cantidad de intervenciones quelos estudiantes efectuaron en la plataforma utilizando las herramientas de comunicación que la misma proporciona. Se consideraron como indicadores la cantidad de intervenciones registradas mediante uso de las herramientas e-mail (EMAIL) y chat (CHAT). Para EMAIL se consideraron los siguientes rangos: o a 10 mails enviados, 11 a 30 mails enviados y más de 30 mails enviados. Para CHAT los rangos considerados fueron: o a 50 contribuciones, 51 a 100 contribuciones y más de 100 contribuciones. A fin de poder asignar valor a la variable NCC se establecieron relaciones entre ella y sus indicadores (Tabla 1).

La variable NPC permitió evaluar la calidad de las contribuciones efectuadas por los estudiantes en tres foros de discusión electrónica (ACT1, ACT2, y ACT3). Para evaluar estas actividades se consideró una escala cualitativa con las siguientes calificaciones posibles: alto (A), medio (M) y bajo (B). A fin de poder asignar valor a la variable NPC se establecieron relaciones entre ella y sus indicadores (Tabla 1).

En la Tabla 2 se muestran los valores que tomaron los indicadores en la experiencia realizada con los estudiantes de la cohorte 2008. 
Finalmente en la Tabla 3, se muestra el valor asignado a las cuatro variables bajo estudio, en función de los valores adoptados por los indicadores (Tabla 2) y las relaciones definidas en Tabla 1.

\begin{tabular}{|c|c|c|}
\hline Var. & Valor & Relación con indicadores cambiar \\
\hline \multirow{3}{*}{ NCT } & Alto (A) & PAR entre 8 y 10 and TALL = A \\
\hline & Medio (M) & $\begin{array}{l}\text { PAR entre } 8 \text { y } 10 \text { and TALL = M } \\
\text { PAR entre } 5 \text { y } 7 \text { and TALL = A } \\
\text { PAR entre } 8 \text { y } 10 \text { and TALL = B } \\
\text { PAR entre } 1 \text { y } 4 \text { and TA1LL = A } \\
\text { PAR entre } 5 \text { y } 7 \text { and TALL = M }\end{array}$ \\
\hline & Bajo (B) & $\begin{array}{l}\text { PAR entre } 5 \text { y } 7 \text { and TALL }=B \\
\text { PAR entre } 1 \text { y } 4 \text { and TALL }=M \\
\text { PAR entre } 1 \text { y } 4 \text { and TALL }=B\end{array}$ \\
\hline \multirow{3}{*}{ NPP } & Alto $(A)$ & $\mathrm{CP}>80$ \\
\hline & Medio (M) & CP entre 61 y 80 \\
\hline & Bajo (B) & CP entre 0 y 60 \\
\hline \multirow{3}{*}{ NCC } & Alto $(A)$ & EMAIL $>30$ and $\mathrm{CHAT}>100$ \\
\hline & Medio (M) & $\begin{array}{l}\text { EMAIL }>30 \text { and CHAT entre } 51 \text { y } 100 \\
\text { EMAIL >30 and CHAT entre } 0 \text { y } 50 \\
\text { EMAIL entre } 11 \text { y } 30 \text { and CHAT > } 100 \\
\text { EMAIL entre } 11 \text { y } 30 \text { and CHAT entre } 51 \text { y } 100 \\
\text { EMAIL entre } 0 \text { y } 10 \text { and CHAT >100 }\end{array}$ \\
\hline & Bajo (B) & $\begin{array}{l}\text { EMAIL entre } 11 \text { y } 30 \text { and CHAT entre } 0 \text { y } 50 \\
\text { EMAIL entre } 0 \text { y } 10 \text { and CHAT entre } 51 \text { y } 100 \\
\text { EMAIL entre } 0 \text { y } 10 \text { and CHAT entre } 0 \text { y } 50\end{array}$ \\
\hline \multirow[b]{2}{*}{ NPC } & Alto (A) & $\mathrm{ACT} 1=\mathrm{A}, \mathrm{ACT} 2=\mathrm{A}, \mathrm{ACT} 3=\mathrm{A}$ \\
\hline & Medio (M) & 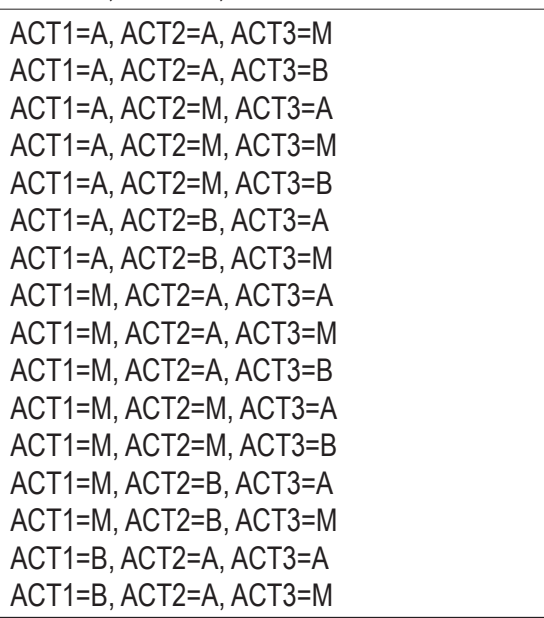 \\
\hline
\end{tabular}


E. Durán; R. Costaguta; M. Gola

El Modelo B-LEARNing ImPLEMENTAdo EN LA AsignAtura Simulación

\begin{tabular}{|c|c|c|}
\hline Var. & Valor & Relación con indicadores cambiar \\
\hline & & $\begin{array}{l}\text { ACT1=B, ACT2=A, ACT3=B } \\
\text { ACT1=B, ACT2=M, ACT3=A } \\
\text { ACT1=B, ACT2=M, ACT3=B }\end{array}$ \\
\hline & Bajo (B) & $\begin{array}{l}\text { ACT1=A, ACT2=B, ACT3=B } \\
\text { ACT1=B, ACT2=A, ACT3=B } \\
\text { ACT1=B, ACT2=B, ACT3=A } \\
\text { ACT1=M, ACT2=B, ACT3=B } \\
\text { ACT1=B, ACT2=B, ACT3=M } \\
\text { ACT1=B, ACT2=M, ACT3=B } \\
\text { ACT1=B, ACT2=B, ACT3=B }\end{array}$ \\
\hline
\end{tabular}

Tabla 1. Vinculación entre las variables e indicadores

\begin{tabular}{|c|c|c|}
\hline Indicador & Valores observados & Valor asumido \\
\hline \multirow{3}{*}{ PAR } & $1-4(16 \%)$ & \multirow{3}{*}{$8-.10$} \\
\hline & $5-7(36 \%)$ & \\
\hline & $8-10(48 \%)$ & \\
\hline \multirow{3}{*}{ TALL } & $\mathrm{A}(38 \%)$ & \multirow{3}{*}{ M } \\
\hline & $M(53 \%)$ & \\
\hline & $\mathrm{B}(9 \%)$ & \\
\hline \multirow{3}{*}{ ACT1 } & $\mathrm{A}(17 \%)$ & \multirow{3}{*}{ B } \\
\hline & $M(17 \%)$ & \\
\hline & $\mathrm{B}(66 \%)$ & \\
\hline \multirow{3}{*}{ ACT2 } & $\mathrm{A}(29 \%)$ & \multirow{3}{*}{ M } \\
\hline & $\mathrm{M}(47 \%)$ & \\
\hline & $\mathrm{B}(24 \%)$ & \\
\hline \multirow{3}{*}{ АСТ3 } & $\mathrm{A}(65 \%)$ & \multirow{3}{*}{ A } \\
\hline & M (24 \%) & \\
\hline & $\mathrm{B}(11 \%)$ & \\
\hline \multirow{3}{*}{$\mathrm{CP}$} & Más de $80(59 \%)$ & \multirow{3}{*}{ Más de 80} \\
\hline & Entre 61 y $80(0 \%)$ & \\
\hline & Entre 0 y $60(41 \%)$ & \\
\hline \multirow{3}{*}{ EMAIL } & Más de $30(0 \%)$ & \multirow{3}{*}{ Entre 0 y 10} \\
\hline & Entre 11 y $30(0 \%)$ & \\
\hline & Entre 0 y $10(100 \%)$ & \\
\hline \multirow{3}{*}{ CHAT } & Más de 100 (0 \%) & \multirow{3}{*}{ Entre 0 y 50} \\
\hline & Entre 51 y 100 (6\%) & \\
\hline & Entre 0 y 50 (94 \%) & \\
\hline
\end{tabular}


E. Durán; R. Costaguta; M. Gola

El Modelo B-LEARNing ImPLEMENTADo EN la Asignatura Simulación

Tabla 2. Indicadores y valores asumidos en la experiencia

\begin{tabular}{|c|c|}
\hline Variable & Valor asignado \\
\hline NCT & $M$ \\
\hline NPP & $A$ \\
\hline NCC & $B$ \\
\hline NPC & $M$ \\
\hline
\end{tabular}

Tabla 3. Valores finales

Los resultados obtenidos en la experiencia muestran que de las cuatro variables consideradas, sólo una de ellas - NCC (Nivel de Comunicación) - resultó con un valor "Bajo". Al respecto, es importante aclarar que una de las principales causas del valor asumido por esta variable se debe a los inconvenientes que tuvieron los estudiantes para hacer uso de la herramienta Chat. Para utilizar la misma, y por una restricción de la plataforma, los estudiantes debían instalar una máquina virtual de Java en la PC desde la que pretendían usar el Chat; requisito que por cuestiones de configuración del equipo, en muchos casos no pudieron satisfacer, y por lo tanto, les fue imposible utilizar dicha herramienta de comunicación. En relación a las otras tres variables (NCT, NPP y NPC), las mismas registraron valores "Medio" y "Alto". A esto se debe sumar como un aspecto positivo, las opiniones favorables manifestadas por los estudiantes en la encuesta, en relación al uso de la plataforma y al grado de contribución de la experiencia en la apropiación de nuevos conocimientos. La encuesta realizada posibilitó recabar las opiniones de 14 estudiantes sobre un total de 17 , lo cual representa el $82.35 \%$ de la población involucrada en la experiencia. Los

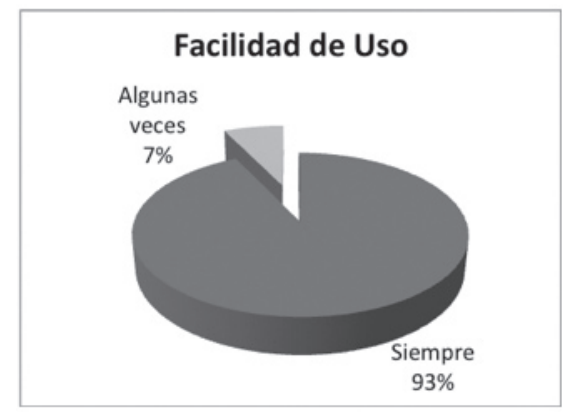

resultados del procesamiento de tales opiniones se muestran en las Figuras 1 a 4 . 


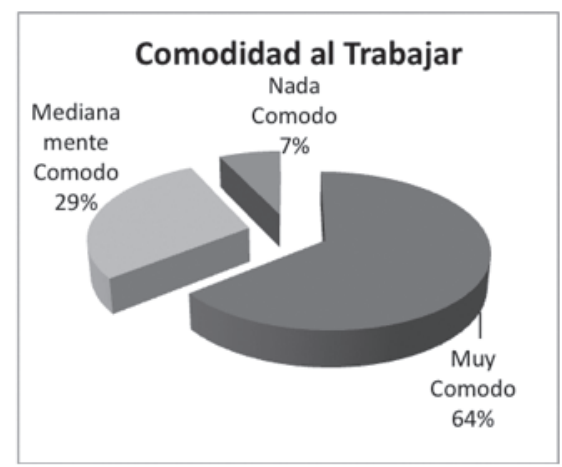

Figura 1. Opinión sobre facilidad de uso de la plataforma

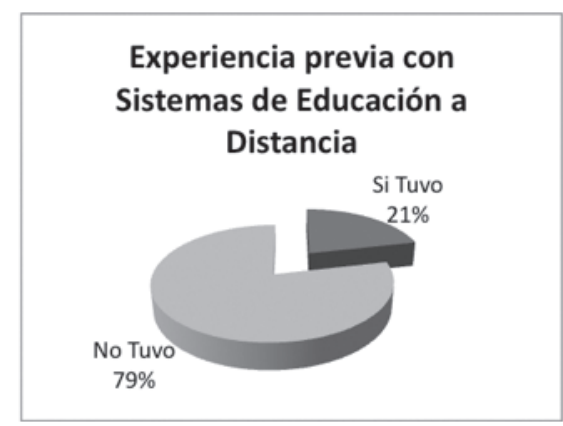

Figura 2. Opinión sobre comodidad para trabajar con la plataforma

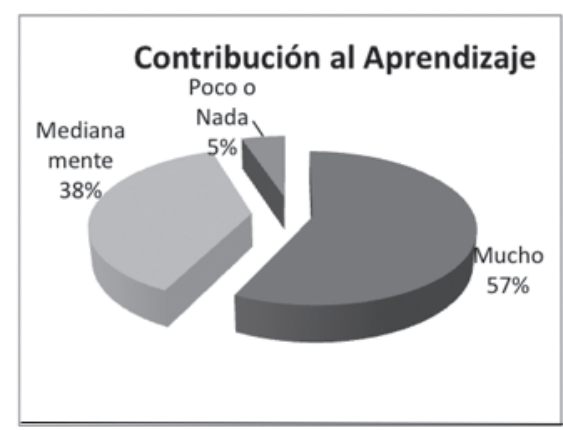

Figura 3. Opinión sobre experiencias con sistemas de aprendizaje a distancia Figura 4. Opinión sobre la contribución al aprendizaje en el trabajo con la plataforma

Con el fin de efectuar un análisis comparativo entre los resultados obtenidos en este trabajo, y los documentados en otras investigaciones realizadas sobre aprendizaje 
mezclado, se llevó a cabo una revisión y análisis de antecedentes. Producto de esta revisión se encontraron varios trabajos que documentan experiencias de aprendizaje mezclado. Por ejemplo, en Bonakdarian et al. (2010) y en Whittaker y Bonakdarian (2011), los autores realizan una experimentación para comparar efectividad, compromiso y eficiencia entre los tres modos de enseñanza (presencial, on-line puro y b-learning). En García y Ponce (2010) se documenta una experiencia de blearning con estudiantes pertenecientes al Profesorado en Computación, pero no se cuantifican resultados. En Santos Bremer et al. (2009) se describe una experiencia realizada con docentes en ciencias, pero no se documentan los resultados obtenidos. En Lamas et al. (2010) se describe una experiencia b-learning universitaria utilizando Moodle, en la que los autores encuestaron a los estudiantes para evaluar la experiencia. En Fainholc (2008) se presenta una experiencia de b-learning a nivel de grado universitario, donde se evalúa la efectividad del aprendizaje logrado por los estudiantes, la satisfacción de los profesores, la satisfacción de los estudiantes, el compromiso institucional, y la relación costo-beneficio de la propuesta; sin embargo, la autora sólo reporta resultados "buenos" en más del 80 \% de los casos para diferentes aspectos que comprende la ficha de "autoevaluación formativa continua"; y una evaluación de la producción de los estudiantes en categoría "buena” en más de un 80\% de los casos, sin aportar detalles respecto a indicadores. En Ruíz Bolivar (2008) se describe una experiencia b-learning en un curso de doctorado, donde el autor obtuvo resultados que indican un alto desempeño académico, una percepción positiva de la calidad del curso, satisfacción de los estudiantes con la experiencia y también una opinión favorable respecto a la modalidad b-learning del curso; sin embargo, no reporta mayores detalles de la evaluación realizada. En Levin-Pedel et al. (2007) se evalúan la comprensión de contenidos, el diseño de actividades y la calidad de la instrucción en tres cursos de b-learning, indicando como resultado que las calificaciones fueron altas en más de un 80\%. En Mariño y López (2007) se describe una experiencia b-learning en asignatura de grado en la que la satisfacción de los estudiantes se evalúa por medio de una encuesta.

En todos los trabajos citados los autores no explicitan los indicadores tomados para evaluar las variables consideradas en el análisis cuantitativo de los resultados, lo cual imposibilitó realizar la comparación planteada. El único trabajo con mayores detalles respecto a los indicadores considerados es el de Bustos Sánchez (2004, 2005), donde se analizan cantidad de accesos o logueos en relación a participación en las clases presenciales, uso de las herramientas que ofrecía la plataforma; cantidad promedio de posteos por estudiante a foros; número de interacciones en debates iniciados por el docente del curso, y en debates iniciados por otros estudiantes, número de accesos a material subido para consulta, uso del recurso bitácora (chat con 
el docente). Comparando estos resultados con los obtenidos en nuestra investigación, se destaca que en nuestra experiencia los estudiantes no utilizaron prácticamente chat y e-mail, cosa que no ocurrió en el trabajo antes citado, donde se utilizaron todas las herramientas ofrecidas por la plataforma. Por otra parte, en el trabajo de Bustos Sánchez se reporta un promedio de 8,7 posteos por alumno en el foro, siendo en nuestro caso de más de 80 contribuciones, lo que denota una participación altamente superior en nuestra experiencia. No obstante, las circunstancias diferentes de ambas experiencias (una fue un curso de extensión, dirigido a formadores, de 30 días de duración, cuyo objetivo era conocer las herramientas en línea de la plataforma; y la otra fue un curso curricular obligatorio, dirigido a estudiantes, cuyo objetivo era adquirir competencias en simulación de sistemas continuos) es lo que justifica fundamentalmente la diversidad de resultados.

\section{DESCRIPCIÓN DE LA EXPERIENCIA COMBINANDO B-LEARNING CON CSCL}

El aprendizaje colaborativo se refiere a un método instruccional en el que los estudiantes trabajan juntos en pequeños grupos para alcanzar una meta común. La inclusión de medios computacionales en el dominio del aprendizaje colaborativo ha permitido definir nuevos escenarios de enseñanza-aprendizaje, originando los conocidos sistemas de CSCL. Estos sistemas ofrecen versiones electrónicas de muchas actividades y recursos presentes en las aulas de enseñanza tradicional (presencial). Contando también con herramientas de comunicación síncrona y/o asíncrona como chat, foro y e-mail, todo lo cual da soporte tanto a la comunicación como a la colaboración entre los estudiantes. El enfoque de CSCL se centra en las dinámicas de grupo y en las actividades basadas en grupo para facilitar el aprendizaje. Sin embargo, constituir un grupo no es suficiente para que los alumnos colaboren. Una colaboración efectiva involucra un conjunto de habilidades que deben ser aprendidas y cultivadas por los estudiantes. Estas adquieren especial importancia en ambientes virtuales donde los alumnos no pueden encontrarse cara a cara (Napier, 2001).

Los estudiantes construyen su aprendizaje colaborativamente manifestando ciertas actitudes, como: explicar y justificar sus opiniones, articular su razonamiento, elaborar y reflexionar sobre sus conocimientos, incentivar a sus compañeros a responder, etc.; actitudes que tienden a motivar y acrecentar el aprendizaje (Soller, 2001). La aparición de estos comportamientos está condicionada, entre otros factores, por las habilidades colaborativas que los estudiantes del grupo sean capaces de manifestar. Soller (2001) propone clasificar las habilidades de colaboración en tres tipos principales: aprendizaje activo, conflicto creativo y conversación. Para 
cada una de estas habilidades establece subhabilidades, y a su vez para cada una de ellas, atributos que las describen, y aperturas de sentencia que los estudiantes suelen utilizar con frecuencia en sus diálogos (Tabla 4).

\begin{tabular}{|c|c|c|c|}
\hline Habilidad & Sub Habilidad & Atributos & Aperturas de Sentencia \\
\hline \multirow{9}{*}{$\begin{array}{l}\text { Conflicto } \\
\text { Creativo }\end{array}$} & Mediación (Med) & Mediación docente & "Preguntémosle al profesor" \\
\hline & \multirow{8}{*}{$\begin{array}{l}\text { Argumentación } \\
\text { (Arg) }\end{array}$} & Conciliar & "Ambos están correctos en eso" \\
\hline & & Concertar & "Yo estoy de acuerdo porque..." \\
\hline & & Discrepar & "Yo no estoy de acuerdo porque..." \\
\hline & & Ofrecer alternativa & "Alternativamente..." \\
\hline & & Inferir & "Entonces...", "Por lo tanto..." \\
\hline & & Suponer & "Si... entonces..." \\
\hline & & Proponer excepciones & "Pero..." \\
\hline & & Dudar & "Yo no estoy seguro" \\
\hline \multirow{15}{*}{$\begin{array}{l}\text { Aprendizaje } \\
\text { activo }\end{array}$} & \multirow{2}{*}{$\begin{array}{l}\text { Motivar } \\
\text { (Mot) }\end{array}$} & Animar & "Muy Bien", "Buen Punto" \\
\hline & & Reforzar & "Está correcto" \\
\hline & \multirow{7}{*}{$\begin{array}{l}\text { Informar } \\
\text { (Inf) }\end{array}$} & Parafrasear & "En otras palabras..." \\
\hline & & Guiar & "Yo pienso que deberían..." \\
\hline & & Sugerir & "Yo pienso" \\
\hline & & Elaborar & "Para elaborar" "Además..." \\
\hline & & Explicar/Clarificar & "Permítanme explicarlo de esta manera...." \\
\hline & & Justificar & "Para justificar..." \\
\hline & & Afirmar & "Yo estoy razonablemente seguro..." \\
\hline & \multirow{6}{*}{$\begin{array}{l}\text { Requerir } \\
\text { (Req) }\end{array}$} & Información & "¿Sabes tú...?" \\
\hline & & Elaboración & ¿Puedes decirme más? \\
\hline & & Clarificación & “¿Puedes explicarme cómo/por qué?” \\
\hline & & Justificación & ¿Por qué piensas eso?” \\
\hline & & Opinión & “¿Piensas tú...?” \\
\hline & & Ilustración & "¿Por favor muéstrame?" \\
\hline \multirow{12}{*}{ Conversación } & \multirow{3}{*}{$\begin{array}{l}\text { Reconocimiento } \\
\quad(\operatorname{Rec})\end{array}$} & Apreciación & "Gracias" \\
\hline & & $\begin{array}{l}\text { Aceptación/ } \\
\text { Confirmación }\end{array}$ & "Bien" "Sí" \\
\hline & & Rechazo & "No" \\
\hline & \multirow{5}{*}{$\begin{array}{l}\text { Mantenimiento } \\
\text { (Man) }\end{array}$} & Requerir atención & "Discúlpenme" \\
\hline & & Sugerir acción & "¿Podrías por favor....?" \\
\hline & & Requerir confirmación & “¿Esta bien?” ¿¿Es esto correcto?” \\
\hline & & Atender & "Yo comprendo lo que estas diciendo" \\
\hline & & Disculparse & "Discúlpame" \\
\hline & \multirow{4}{*}{ Tarea (Tar) } & $\begin{array}{l}\text { Coordinar procesos } \\
\text { grupales }\end{array}$ & $\begin{array}{l}\text { "Bien. Continuemos", “¿Están ustedes } \\
\text { listos?" }\end{array}$ \\
\hline & & $\begin{array}{l}\text { Requerir cambio de } \\
\text { enfoque }\end{array}$ & "Permítanme mostrarles..." \\
\hline & & Resumir información & "Para resumir" \\
\hline & & Finalizar participación & "Adios" \\
\hline
\end{tabular}


Tabla 4. Taxonomía de Habilidades Colaborativas

Para lograr una colaboración efectiva es preciso conocer cuáles de estas habilidades poseen los alumnos intervinientes en una comunidad de aprendizaje y, cuáles son aquellas de las que carecen. Con ese fin se realizó la segunda experiencia, en la que se combinó el modelo b-learning con el aprendizaje colaborativo; buscando indagar además la incidencia de ambos modelos en la calidad del proceso de enseñanza- aprendizaje en la asignatura Simulación. Dicha experiencia se llevó a cabo con la cohorte 2009 de la mencionada asignatura, conformada por 13 estudiantes, y se centró en el abordaje de las dos últimas unidades temáticas del programa de estudio de esta asignatura: Realidad Virtual (Unidad 6) y Nuevas tendencias de la Simulación (Unidad 7). La variante introducida respecto a la experiencia anterior fue la implementación de actividades para ser resueltas colaborativamente por los estudiantes, utilizando foros grupales en la plataforma de e-learning.

Para identificar las habilidades de colaboración manifestadas por cada estudiante, se analizaron los textos de los foros, reconociendo en ellos las aperturas de sentencia sugeridas por Soller en la tabla 4, y etiquetando cada intervención con un atributo de la mencionada tabla. Luego, se totalizaron las intervenciones etiquetadas por sub-habilidad. Así por ejemplo, la intervención del estudiante x que expresa "Yo pienso que sería conveniente comenzar por la actividad 2...”, fue etiquetada como Sugerir y por lo tanto contabilizada en la sub-habilidad INFORMAR.

\section{RESULTADOS OBTENIDOS EN LA EXPERIENCIA COMBINANDO B- LEARNING CON CSCL}

En esta experiencia se evaluaron idénticas variables que en el caso anterior, obteniendo resultados similares. Pero además, se recabó información respecto a las habilidades de colaboración manifestadas por los estudiantes. En la Figura 5 se muestra el porcentaje en el que se manifestó cada tipo de habilidad en el total de los alumnos.

Estos resultados muestran que las sub-habilidades de colaboración que manifestaron en mayor medida los estudiantes en sus trabajos, fueron la subhabilidad de Informar (Inf) en un 41,16\% y la sub-habilidad de Argumentar (Arg) en un 22,11\%; mientras que las que no se manifestaron o estuvieron presentes en porcentajes muy bajos fueron: Mediar (Med) y Motivar (Mot). Así, quedó de manifiesto que las habilidades más desarrolladas en los estudiantes son las que requieren de un protagonismo individualista por parte de los mismos, y las menos desarrolladas son aquellas que requieren de la colaboración entre pares. 
Probablemente, esto tenga vinculación, en parte, con la falta de experiencia previa de los estudiantes en participar de este tipo de experiencia, ya que según refleja la encuesta para el 79\% de los estudiantes era su primer trabajo de estas características. Si bien las habilidades colaboración más desarrolladas en el grupo de estudiantes participantes de la experiencia fueron Informar y Argumentar, que son habilidades más individualistas, es muy rescatable el interés de los estudiantes por participar en las discusiones de los foros, ya que como lo muestra la variable $\mathrm{CP}$, el 59\% de los estudiantes tuvieron más de 80 intervenciones. No obstante, será necesario diseñar a futuro actividades tendientes a desarrollar en los estudiantes la capacidad de mediar ante diferencias dentro de los grupos de trabajo, y de motivar a sus compañeros en la elaboración de la tarea, como así también, se deberá trabajar en el desarrollo de las subhabilidades correspondientes a la categoría "conversación", que les permita mejorar la participación en los grupos de aprendizaje colaborativo a distancia en los

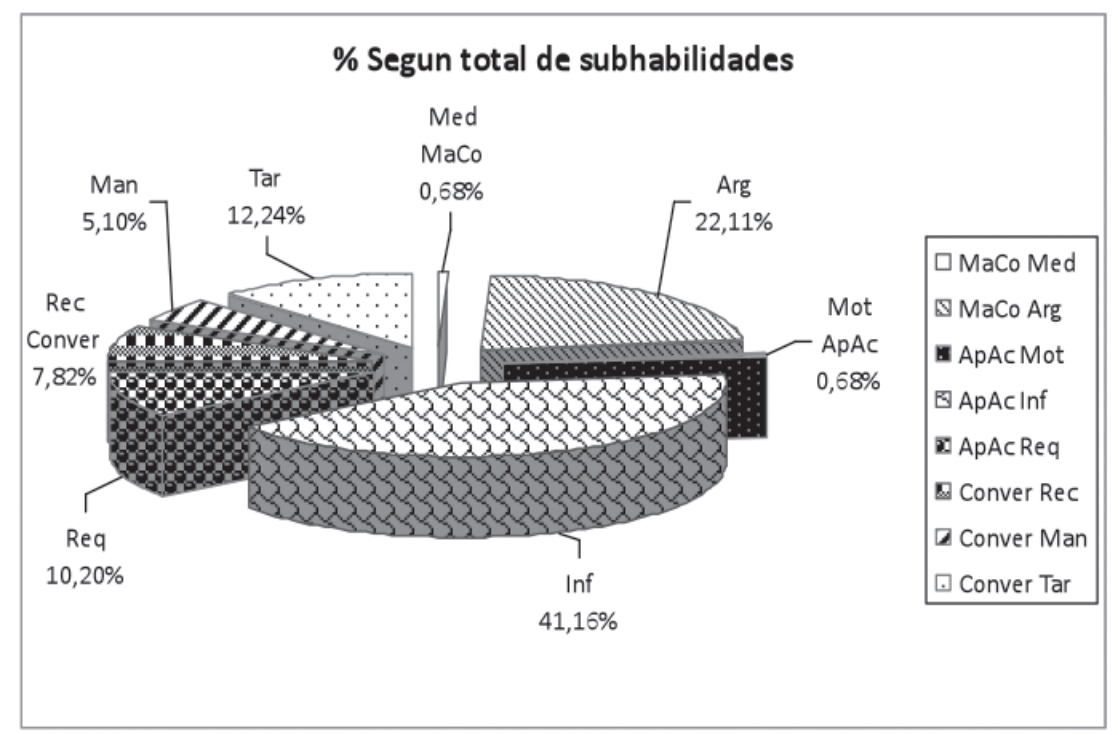

que participen.

Figura 5. Manifestación de habilidades colaborativas en los estudiantes

\section{CONCLUSIONES}

Del análisis de los resultados obtenidos en ambas experiencias se puede concluir que la implementación del modelo b-learning en la asignatura Simulación, para los estudiantes de Ingeniería en Computación de las cohortes 2008, fue positiva; como así también para la cohorte 2009, en la que el modelo b-learning se combinó con el 
aprendizaje colaborativo.

Por otra parte, se rescata la importancia del estudio realizado en cuanto al análisis detallado de cada una de las variables involucradas, especialmente por los pocos antecedentes encontrados que reportan detalles sobre los indicadores utilizados en los estudios realizados. Además, no hay registros de investigaciones sobre habilidades de colaboración demostradas por estudiantes en grupos de aprendizaje colaborativo en entornos de e-learning, lo que destaca la originalidad del estudio realizado.

Los resultados obtenidos permiten afirmar que se lograron procesos de enseñanza y de aprendizaje de calidad en la asignatura Simulación, al haber combinado el modelo b-learning con el aprendizaje colaborativo, y que por lo tanto, ambos enfoques deben ser considerados como una alternativa válida en las planificaciones futuras de esta asignatura y de otras con características similares.

\section{REFERENCIAS BIBLIOGRÁFICAS}

Aballay, A.; Carestia, N.; Martín, A. (2006). Implementación de una Unidad Didáctica Utilizando la Plataforma educativa Exactas a Distancia. Revista Iberoamericana de Tecnología en Educación y Educación en Tecnología, 1 .

Bartolomé, A. (2004). Blended Learning. Conceptos Básicos. Pixel-Bit Revista de medios y Educación, 23.

Bonakdarian, E.; Whittaker T.; Yan, Y. (2010). Mixing it up - more experiments in hybrid learning. Journal of Computing Sciences in Colleges, 25 (4).

Bustos Sánchez, A. (2004). Un modelo para blended-learning. Un caso aplicado a la formación en el trabajo. Compartimos prácticas ¿compartimos saberes? Revista Iberoamericana de Educación a Distancia, 7 (1 y 2).

Bustos Sánchez, A. (2005). Un modelo para blended-learning. Un caso aplicado a la formación en el trabajo. Virtual Educa, México.

Fainholc, B. (2008). Modelo tecnológico en línea de Aprendizaje electrónico mixto (o Blended learning) para el desarrollo profesional docente de estudiantes en formación, con énfasis en el trabajo colaborativo virtual. Revista de Educación a Distancia, 21.

Feierherd, G.; Depetris, B.; De Giusti, A. (2005). Una experiencia de Blended Learning en la asignatura Sistemas Distribuidos en la Sede Ushuaia de la UNPSJB, en Proc. Primeras Jornadas de Educación en Informática y TICs en Argentina.

García, B.; Ponce, V. (2010). BlendedLearning una Experiencia de Enseñanza y Aprendizaje para la Licenciatura en Comunicación Social. En Proc. 3er Congreso Virtual Iberoamericano de Calidad en Educación a Distancia.

Gonzalez Mariño, J. (2006). B-Learning utilizando software libre, una alternativa viable en Educación Superior. Revista Complutense de Educación, 17 (1).

Kowalski, V.; Erck, I.; Molina, S.; Hedman, G. (2008). B-Learning en la formación de ingenieros industriales: una experiencia educativa en el dictado de Investigación Operativa, en Proc. VI Congreso Argentina de Enseñanza de la Ingeniería.

Lamas, M. L.; Massié, A. I.; Quero, E. D. (2010). Implementación de un aula 
virtual bajo la modalidad mixta: El Caso de Química Agrícola en la Universidad Nacional de Salta. Revista Formación Universitaria, 3 (4).

Levin-Peled, R.; Kali, Y.; Judy Dori, Y. (2007). Promoting Collaborative Learning in Higher Education: Design Principles for Hybrid Courses. En Proc. 8th International Conference on Computer Supported Collaborative Learning (CSCL).

López, A.; Azcárate, V. (2006). Experiencias innovadoras de formación combinada (b-learning). En Proc. Congreso de la Cibersociedad 2006. Eje temático D: Educación y Aprendizaje. D-8. Las tecnologías digitales y la producción colaborativa de contenidos educativos.

Mariño, S. I.; López, M.V. (2007). Aplicación del modelo b-learning en la asignatura "Modelos y Simulación" de las carreras de Sistemas de la FACENA-UNNE. Revista Electrónica de Tecnología Educativa (Edutec), 23.

Napier, W.; Waters, L. (2001). Building Team Collaboration in the Virtual Classroom, en Proceedings for Association for educational Communications and Technology (AECT) International Convention: "Leadership and Technology", 1, (303-311).

Peña, A. (2005). Collaborative Student Modeling by Cognitive Maps, en Proceedings of the First International Conference on Distributed Frameworks forMultimediaApplications(DFMA' $\left.{ }^{\circ} 5\right)$. IEEE Press.

Rosas, P. (2005). La Gestión de Ambientes Virtuales de Aprendizaje en los Postgrados de la Universidad de
Guadalajara, en Proc. Tecnologías para Internacionalizar el Aprendizaje.

Ruíz Bolívar, C. (2008). El Blended-Learning: Evaluación de una Experiencia de Aprendizaje en el Nivel de Posgrado. Revista Investigación y Posgrado, 23 (1). Universidad Pedagógica Experimental Libertador, Venezuela.

Sánchez-Cortés, R.; García Manso, A.; Sánchez Allende, J.; Moreno Díaz, P.; Reinoso Peinado, A. (2005). B-Learning y Teoría del Aprendizaje Constructivista en las Disciplinas Informáticas: Un esquema de ejemplo a aplicar. Recent Research Developments in Learning Technologies.

Santos Bremer, A. D.; Gil de Marrupe, M.; Valdés, S. P. (2009). La Plataforma Virtual en la Formación Continua en Ciencias Biológicas: Una Experiencia con Docentes del Nivel Secundario. Revista Electrónica Iberoamericana de Educación en Ciencias y Tecnología, 1 (1).

Sanz, C.; Madoz, C.; Gorga, G.; Gonzalez, A. (2008). La importancia de la modalidad 'blended learning'. Análisis de una experiencia educativa. Revista Iberoamericana de Tecnología en Educación y Educación en Tecnología, 3.

Soller,A.(2001).SupportingSocialInteraction in an Intelligent Collaborative Learning System. En International Journal of Artificial Intelligence in Education (2001), 12.

Whittaker, T.; Bonakdarian, E. (2011). Faceto-face experiences for online students: effective, efficient, and engaging hybrid classes. Journal of Computing Sciences 
in Colleges, 26 (4).

\section{PERFIL ACADÉMICO Y PROFESIONAL DE LOS AUTORES}

Elena Durán. Doctora en Ciencias de la Computación. Docente e investigadora de la Universidad Católica y de la Universidad Nacional de Santiago del Estero, Argentina.

E-mail: elena.duran@ucse.edu.ar

Rosanna Costaguta. Doctora en Ciencias de la Computación. Docente e investigadora de la Universidad Católica y de la Universidad Nacional de Santiago del Estero, Argentina.

E-mail: rcostaguta@hotmail.com

Mariela Gola. Ingeniera en Computación. Docente e investigadora de la Facultad de Matemática Aplicada, Universidad Católica de Santiago del Estero.

E-mail: Mariela.gola@ucse.edu.ar

\section{DIRECCIÓN DE LAS AUTORAS}

Facultad de Matemática Aplicada

Universidad Católica de Santiago del Estero, Av. Alsina (E) y Dalmacio Vélez Sársfield Santiago del Estero

Argentina

Fecha de recepción del artículo: 04/03/2011

Fecha de aceptación del artículo: 04/05/2011

\section{Como citar este artículo:}

Durán, E.; Costaguta, R.; Gola, M. (2011). El modelo B-learning implementado en la asignatura simulación. RIED. Revista Iberoamericana de Educación a Distancia, volumen 14, $\mathrm{n}^{0}$ 2, pp. 149-166. 